\title{
The simulation of coherent optical communication technology
}

\author{
Mingyang Li ${ }^{1}$, Man Feng ${ }^{2}$,Pengyuan $\mathrm{Chen}^{3}$,Zhenping Lan ${ }^{4}, \operatorname{Ping} \mathrm{Li}(\mathbb{})$ \\ \{ $\underline{523155344 @ q q . c o m}{ }^{1}$, fengman2020@163.com ${ }^{2}, 554855487 @ q q . c^{3}{ }^{3}$, \\ lanzp@dlpu.edu.cn ${ }^{4}$,liping@dlpu.edu.cn-凶-\}
}

Department of Information Science and Engineering Dalian Polytechnic University Dalian, P. R. China

\begin{abstract}
With the advent of the era of big data and the development of Internet technology, various emerging businesses have emerged. Due to the lack of optical coherence, the essence of optical fiber communication is a noise carrier communication system. In order to be able to communicate with high capacity over long distance, various modulation methods and heterodyne reception are used to construct coherent optical communication system. Heterodyne/coherent fiber communication is being accelerated in its research and development due to the possibility of increasing the receiving sensitivity by $20-30 d B^{[1]}$. The coherent optical communication system has good selectivity and large communication capacity. The receiving sensitivity of the coherent balanced detection technology is about $10 \mathrm{~dB} \sim 20 \mathrm{~dB}$ higher than that of the incoherent optical communication system, which greatly extends the transmission distance ${ }^{[2]}$. This paper will study the optical coherence communication system and its simulation, the main components are as follows:
\end{abstract}

1. General information and importance of the development status and future trend of coherent optical communication.

2. A review of coherent optical communication system.

3. Based on the understanding and analysis of the previous chapter, the OptiSystem software platform is used for system simulation. Designed the core algorithm of DSP processing unit and simulated the coherent optical communication system.

Keywords: Coherent optical communication system; the key technology of coherent optical communication; OptiSystem simulation.

Corresponding author: Ping Li(1969-), female, Professor, master, main research directions for optical communications and intelligence systems.

E-mail:liping@dlpu.edu.cn

Room 312, Photonics Institute, Dalian Polytechnic University, No. 1, Light Industry Garden, Ganjingzi District, Dalian. 


\section{Introduction}

\subsection{Background and significance of the project}

Information is booming rapidly. With the development of the global Internet, people have made great efforts to catch up with the huge increase in global traffic demand ${ }^{[3]}$.Heterodyne detection is the main method to realize information transmission detection by using coherent optical communication technology, which can ensure that communication managers can find out problems such as frequency conversion difference in information transmission, so that managers can modify information transmission parameters and optimize the quality of information transmission.

In the 21st century, there has been an explosive growth of broadband information services, and the existing optical communication systems are facing serious data transmission problems. A communication system is urgently needed to make the information transmission faster, stronger and farther.In recent years, coherent optical communication technology has developed rapidly, because coherent optical communication makes full use of the mixed frequency gain, good channel selectivity and wavelength tunability of coherent communication ${ }^{[4]}$.

\subsection{Development status and future trend of coherent optical communication}

Optical fiber technology is widely used in communication construction. With the extensive use of communication, the application of optical fiber technology is gradually expanding [5].The ultimate goal of scientists is always to increase the transmission bandwidth, increase the transmission distance and improve the reception sensitivity as the research direction of optical fiber communication.In recent years, with the spread of Internet video, the information generated by them has increased dramatically. The application of these communication technologies is huge, which requires the stability and efficiency of the communication system.The transport performance of the physical layer becomes more and more important.Therefore, wave-multiplexing technology no longer meets the requirements of optical fiber communication for transmission distance and bandwidth.Although erbium-doped fiber amplifier (EDFA) is also in use, it still fails to meet the requirements of optical fiber communication researchers [6].The emergence of new technology inevitably requires the emergence of new equipment.Optical equipment has always been expensive, if large-scale replacement of communication equipment.Operators can't afford to spend so much.Therefore, for equipment manufacturers, the development of new optical communication is also risky.We now need to improve the performance of optical communication systems on existing equipment.Coherent optical communication system is born in this case.

\section{A Review of coherent optical communication systems}

\subsection{Basic principles of coherent light detection}

Coherent modulation and heterodyne detection are the main means of coherent optical communication ${ }^{[7]}$.The basic principle is that the signal light first acts on the optical carrier by means of external light modulation, which has various modulation modes, including amplitude, frequency or phase modulation, and then passes through the optical detector.All above belong to the optical transmitter part.The signal is transmitted to the optical fiber, belonging to the optical fiber part.At the receiving end, the signal optical signal is coherently coupled with the local optical oscillator at the mixer, and then the signal is output to the optical detector for 
detection.In order to ensure a good match between the modulated light wave output from the photodetector and the fundamental mode $\mathrm{HE}_{11}$ in the single-mode fiber, and to ensure that the polarization state of the modulated light wave modulated by external light is consistent with the local vibration state in the single-mode fiber, it is necessary to detect at the transmitter part.

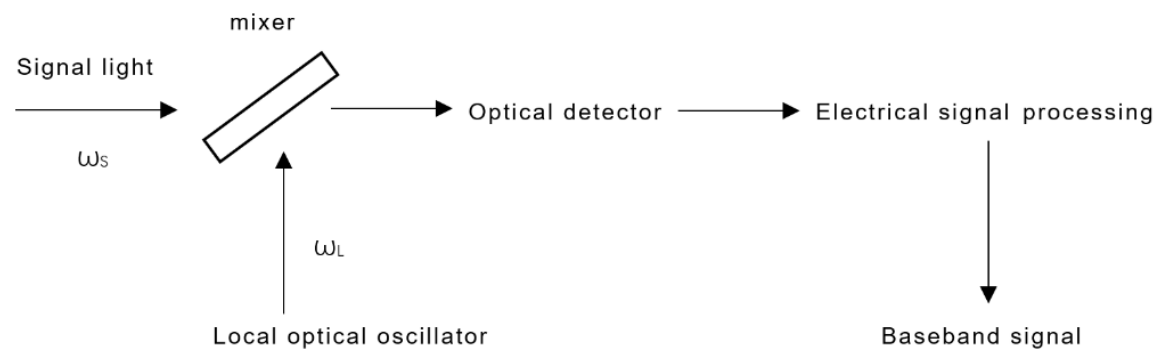

Fig. 1. Principle block diagram of optical coherence detection

\subsection{Composition of coherent optical communication system}

Two beams of light vibrate in the same direction, have the same oscillation frequency, the same phase or phase difference.Such two beams of light meet the conditions of coherence, known as coherent light.Coherent optical communication system produces coherent optical transmitter, coherent optical receiver and intermediate frequency signals generated by optical transmission link.After photoelectric detection and enhanced if signal, the demodulator recovers the output signal required by the user ${ }^{[2]}$.Compared with traditional intensity modulation techniques, the receiver is more sensitive, can maintain a higher modulation level, and can extract all information about electric field light ${ }^{[8]}$.Coherent optical communication system consists of three parts: coherent optical transmitting terminal, optical transmission link and coherent optical receiving terminal.

The coherent optical transmitter receiver includes channel coding, external modulator, automatic bias control circuit of external modulator, narrow line-width laser light source, optical splitter, polarization control, etc ${ }^{[2]}$.The optical transmitter assembly converts the HDB3 signal encoding from a multistage device to an NRZ code, which is converted to the code available on the photoelectric cable, and then converts the electrical signal to an optical signal and enters the optical fiber for communication.

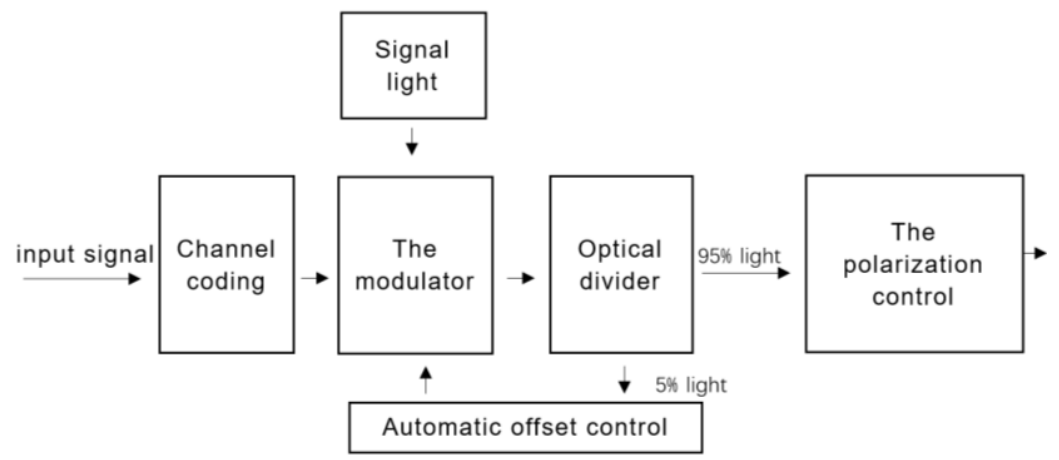

Fig. 2. Coherent optical transmitter 
The function of optical transmission link is to transmit modulated modulated light waves from coherent optical transmitter to coherent optical receiver. The transmission mode is $\mathrm{HE}_{11}$ mode.Attenuation of the amplitude, phase delay and possible change of polarization direction of the light wave occur during the whole transmission process.

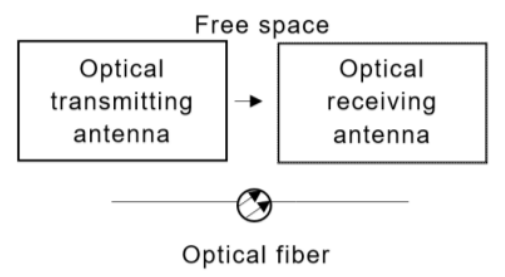

Fig. 3. Optical transmission link

The light transmitted from the optical transmission link goes into a matching device that matches the transmitter.The device also allows receiving the spatial distribution and polarized light state based on the laser's output of its own vibration. The mixer is used to mix the local light wave (frequency $\mathrm{f}_{1}$ ) and the received light wave (frequency $\mathrm{f}_{\mathrm{S}}$ ) with each other, and the photoelectric detector is used for detection, and then the difference frequency signal (frequency $\mathrm{f}_{\mathrm{S}}-\mathrm{f}_{1}$ ) between them is detected and amplified with an intermediate frequency amplifier.

\subsection{Key technology of coherent optical communication}

It mainly includes the following: signal source and local vibration source must use laser with stable frequency and high spectral purity.Ordinary lasers cannot meet this requirement, so it is necessary to study frequency stabilization technology.In order to obtain high mixing efficiency, it is necessary for coherent optical communication system to carry out very strict matching conditions when signal light and local oscillator light are mixed.

If it is directly modulated by optical laser parameters. Then the other parameters will always cause parasitic vibrations, just as the modulation frequency is when the phase changes.In addition, they may also encounter problems such as unstable loose frequencies. Therefore, in addition to the current directly injected modulation frequency, other transmitted light wave modulation adopts external light modulation [14].External light modulation depending on physical phenomena, some characteristics of photocrystals or optical crystals differ from external factors.For example, a diffused LiNbO3 Mach interferometer or a directionally coupled modulator can be used for ASK modulation, and a semiconductor quantum well phase external modulator or LiNbO3 phase modulator can be used for PSK modulation [14].

In coherent optical communication system, coherent control requires the signal source to be polarized in the same direction, that is, the vector direction of two light sources is the same, in order to obtain high coherent reception sensitivity, otherwise the sensitivity of coherent detection will be greatly reduced.Because the polarization direction of the signal light source and the local oscillator light source is the same, only the projection of the electric signal vector can truly generate the intermediate frequency current ${ }^{[14]}$. Receiver sensitivity can be difficult to ensure if the Angle misalignment exceeds $60^{\circ}$, which may lead to the loss of coherent reception advantage. Therefore, in order to make full use of the benefits of coherent reception, measures are being taken to stabilize polarization.

Polarization-maintaining fiber: in order to let the process of optical wavelength polarization state remains unchanged (ordinary single-mode fiber will change the light 
polarization state, reason is due to the mechanical vibration of the fiber or temperature change and other factors), but compared with the single mode fiber, "polarization-maintaining fiber with high loss and price on the high side ${ }^{[14]}$.Polarization classification: The use of a common single-mode fiber, using polarization classification technology at the receiving end of the coherent light.After the signal source is mixed with the local oscillator, it is first divided into two channels for balanced reception.For each channel signal, polarizing beam splitter is used to divide them into orthogonal polarized signals of two channels for detection, and then the sum of squares is calculated.Finally, the two receiving channels are judged, and the better channel is selected as the output signal.However, the output signal is independent of the polarization state of the received signal, which eliminates random variations in the polarization mode of the signal during transmission.

Laser frequency stabilization is very important in coherent optical communication.For example, when the frequency (or wavelength) of the laser changes under different working conditions, it is difficult to determine the zero deviation by the relative frequency stability of the light source in the coherent optical communication system. This also applies to heterodyne coherent optical communication systems.In general, the average heterodyne frequency is between 0.2 and $2 \mathrm{GHz}$.If the optical carrier is $1.5 \mathrm{~m}$, she will have a $200 \mathrm{THz}$ intermediate frequency of $10^{\wedge}(-6) \sim 10^{\wedge}(-5)$.If the optical frequency carrier changes, there will be a large average frequency change.Therefore, it is necessary to ensure the stability of the highfrequency light wave generator and light wave, so that the coherent light communication system can work normally ${ }^{[14]}$.

The amplitude noise of the semiconductor laser and the influence of frequency modulation on the sensitivity of the receiver can be overcome when the narrowband of the light wave is ensured.The narrower the line width is, the smaller the phase shift generated by the noise will be ${ }^{[14]}$. There are two ways to do this :(1) this is a method of blocking patterns.In other words, the intensity of the laser is the stable frequency and narrow spectral line, and the single-type laser introduces the laser with spectral width, similarity and frequency stability, and the laser is mainly the compression width.(2) External cavity feedback method.The external cavity feedback is that the laser output passes through the mirror cavity and then through the external, grating and other dispersed elements, so as to obtain the external cavity with dynamic single-mode application and select the characteristic using the mode, and the width of the compression spectral line depends on the $Q$ value of the external cavity ${ }^{[14]}$.

Coherent optical communication often uses a strong frequency separation method, so nonlinear effects in optical fiber communication may cause the signal strength and phase strength of a single optical communication channel to depend on the conduction frequency of another channel and nonlinear continuous interference.The effect of the nonlinear refractive index fiber, and thus the phase modulation of phase noise, should be considered when the signal power is greater than $10 \mathrm{~mW}$ in coherent optical communication systems or when the signal is transmitted over long distances through optical amplifiers. When the channel space and fiber dispersion are small enough, the phase condition of FWM can be satisfied.FWM is an important component of nonlinear intervention.FWM limits the performance of the system and reduces the energy of the channel to block the channel. When the channel power is less than a certain value, the influence of FWM on the system can be avoided ${ }^{[14]}$.

Signal light and local oscillator light need to be provided by coherent optical communication.It requires narrow band of a longitudinal module, high frequency stability, dynamic single frequency characteristic of dynamic laser signal in dynamic modulation, width of modulation band meeting coding speed requirement.At the same time, it is also necessary to design a good control circuit to make the laser output very narrow line-light stability at 
different temperatures.Narrow and stable spectral line width is a necessary condition for the practical application of coherent optical communication system. The wider the spectral line of the light source, the greater the phase noise and the worse the sensitivity will be. The spectral line width of the light source itself will determine the lowest bit error rate and the highest receiving sensitivity that the system can achieve $\mathrm{e}^{[2]}$.

\section{Simulation of Coherent Optical Communication System Based on OptiSystem}

In this paper, the QPSK coherent optical communication system for local area network (LAN) is simulated.QPSK modulation and demodulation technology, also known as orthographic phase shift keying technology, belongs to a phase modulation technology ${ }^{[9]}$.It is improved by PSK technology, and has many advantages over traditional modulation and demodulation technology. It transmits more information in limited bandwidth, and the interference has little effect on the signal during transmission.

\subsection{Design principle}

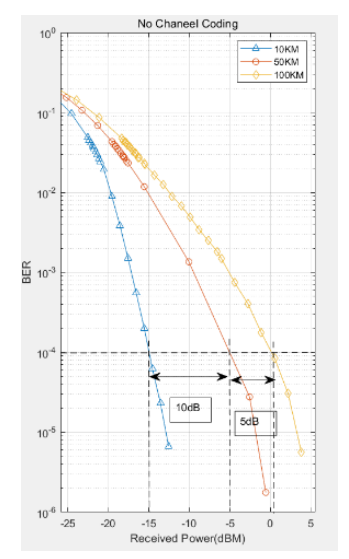

Fig. 4. Design schematic

Figure 4 is the design schematic diagram of QPSK coherent optical communication system.As shown in the figure, the probability of bit error increases with the increase of received power.And the longer the transmission distance, the higher the bit error rate, both of which affect the bit error rate. When bit error rate is $10^{-4}$, transmission distance of $50 \mathrm{~km}$ will reduce $10 \mathrm{~dB}$ from receiving power of $10 \mathrm{~km}$, while transmission distance of $100 \mathrm{~km}$ will reduce $5 \mathrm{~dB}$ from receiving power of $50 \mathrm{~km}$.

\subsection{Circuit design}

Figure 5 is the design drawing of the 1Gbit/s QPSK coherent optical communication system. The pseudo-random binary sequence of $1 \mathrm{Gbit} / \mathrm{s}$ is generated by the pseudo-random sequence generator and input to the QPSK optical transmitter, which sends the modulated signal into the optical transmission link for transmission.Then the QPSK optical receiver demodulates the received signal through coherent detection, then amplifies and filters, and finally recovers the QPSK signal.The performance of the system is evaluated by bit error rate (BER), error vector amplitude percentage, received signal power, and constellation ${ }^{[10]}$. 


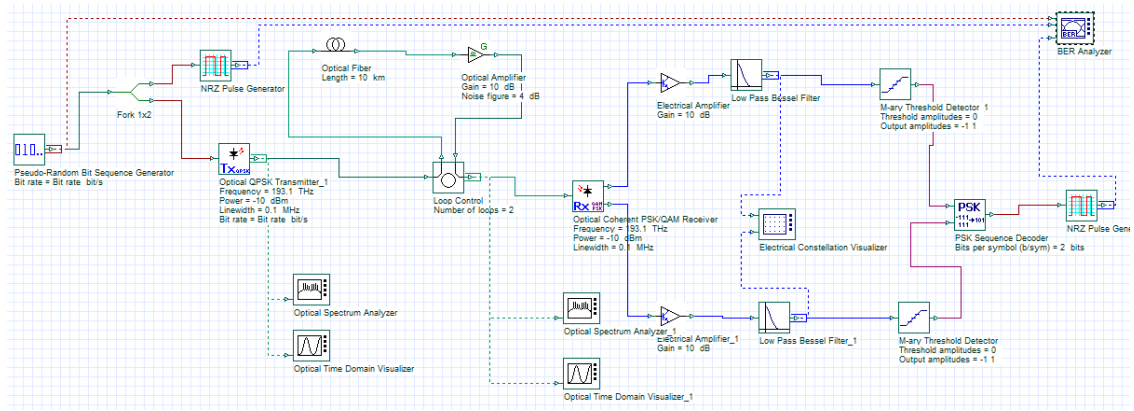

Fig. 5. Circuit diagram

\subsection{The results demonstrate}

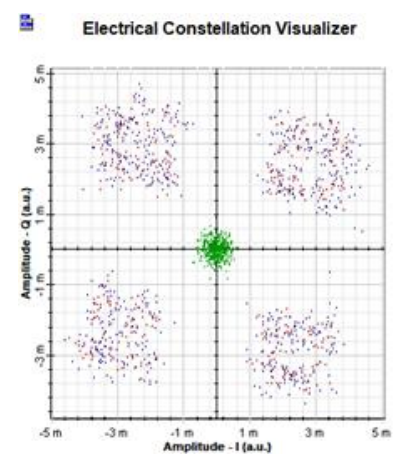

Fig. 6. Result illustration

Figure 6 is the constellation chart of QPSK coherent optical communication system.It can be seen that this constellation chart is not good, appear more obvious constellation blur and deviation.It can be seen from the constellation that the signal is strongly affected by noise and is far away from the ideal vector point with a high error rate.The primary task of communication is to transmit information. Therefore, the main index of communication system quality is the reliability of information transmission.Reliability means the accuracy of information. In digital communication system, the accuracy of information is mainly measured by the frequency in THE BER code. What we need is high reliability and low bit error rate.In order to realize the long distance accurate and stable transmission of optical communication system, we added DSP component to reduce bit error rate. The following is the design of DSP processing unit.

\subsection{DSP processing unit core algorithm design}

The coherent scheme can not only significantly extend the communication distance, but also significantly improve the information carrying capacity ${ }^{[11]}$. The coherent control method uses digital signal processing (DSP) to balance and compensate PMD polarization mode and electric field nonlinear effect, and improve the circuit's resistance to dispersion and ROADM filtering limit ${ }^{[12]}$.

In coherent QPSK systems, when the symbol phase is $\left\{0, \frac{\pi}{2}, \pi, \frac{3 \pi}{2}\right\}$ or $\left\{\frac{\pi}{4}, \frac{3 \pi}{4}, \frac{5 \pi}{4}\right.$, $\left.\frac{7 \pi}{4}\right\}$, the sampling value of the Kth symbol received is assumed to be

$$
\mathrm{S}(\mathrm{k})=\mathrm{e}^{\mathrm{j}\left(\theta_{\mathrm{S}}(\mathrm{k})+\Delta \omega \mathrm{kT}+\theta_{\mathrm{L}}(\mathrm{k})+\theta_{\mathrm{n}}(\mathrm{k})\right)} .
$$




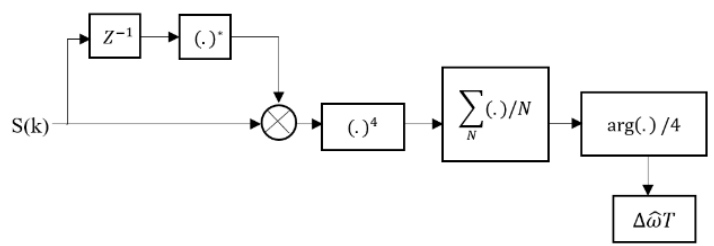

Fig.7.Block diagram of quadrature frequency offset estimation algorithm

The quadriple algorithm's quadratic error assessment process usually consists of four steps: the first step is to eliminate the phase damage $\theta_{\mathrm{L}}$ caused by the laser linewidth.In high-speed optical transmission systems, $\theta_{\mathrm{L}}$ changes slowly relative to the symbol rate, with $\theta_{\mathrm{L}}$ before and after being considered constant.Then, the phase difference between the two symbols can be eliminated by calculating the phase difference

$$
\mathrm{S}(\mathrm{k}) \mathrm{S}^{*}(\mathrm{k}-1)=\mathrm{e}^{\mathrm{j}\left(\Delta \theta_{\mathrm{s}}+\Delta \omega \mathrm{T}+\theta_{\mathrm{n}}\right)} .
$$

The second step is to remove the remaining modulation phase $\Delta \theta_{\mathrm{s}}$. Whether the modulation phase of the QPSK signal is $\left\{0, \frac{\pi}{2}, \pi, \frac{3 \pi}{2}\right\}$ or $\left\{\frac{\pi}{4}, \frac{3 \pi}{4}, \frac{5 \pi}{4}, \frac{7 \pi}{4}\right\}$, the QPSK signal has a value of $\left\{0, \frac{\pi}{2}, \pi, \frac{3 \pi}{2}\right\}$.Through the fourth power operation of the processing result of the first step, the phase component of the removed $\Delta \theta_{\mathrm{s}}$ part is:

$$
\left(\mathrm{S}(\mathrm{k}) \mathrm{S}^{*}(\mathrm{k}-1)\right)^{4}=\mathrm{e}^{\mathrm{j}(4 \Delta \omega \mathrm{T}+4) \theta_{\mathrm{n}}} .
$$

The third step is to remove the noise phase $\theta_{n}$.In high-speed optical transmission system, the deviation between laser frequency and symbol speed is also very slow.The difference produced by frequency deviation from the adjacent $\mathrm{N}$ symbol can be considered as a constant $\Delta \omega$ T.The calculated mean value of $\mathrm{N}$ results can approximately eliminate the influence of noise phase on the estimated results

$$
\sum_{\mathrm{N}} \frac{\left(\mathrm{S}(\mathrm{k}) \mathrm{S}^{*}(\mathrm{k}-1)\right)^{4}}{\mathrm{~N}}=\mathrm{e}^{\mathrm{j} 4 \Delta \widehat{\omega} \mathrm{T}}
$$

The fourth step is to extract carrier frequency offset valuation.

$$
\frac{\arg \left\{\sum_{\mathrm{N}} \frac{\left(\mathrm{s}(\mathrm{k}) \mathrm{S}^{*}(\mathrm{k}-1)\right)^{4}}{\mathrm{~N}}\right\}}{4}=\Delta \widehat{\omega} \mathrm{T} .
$$

\subsection{Circuit design based on DSP}

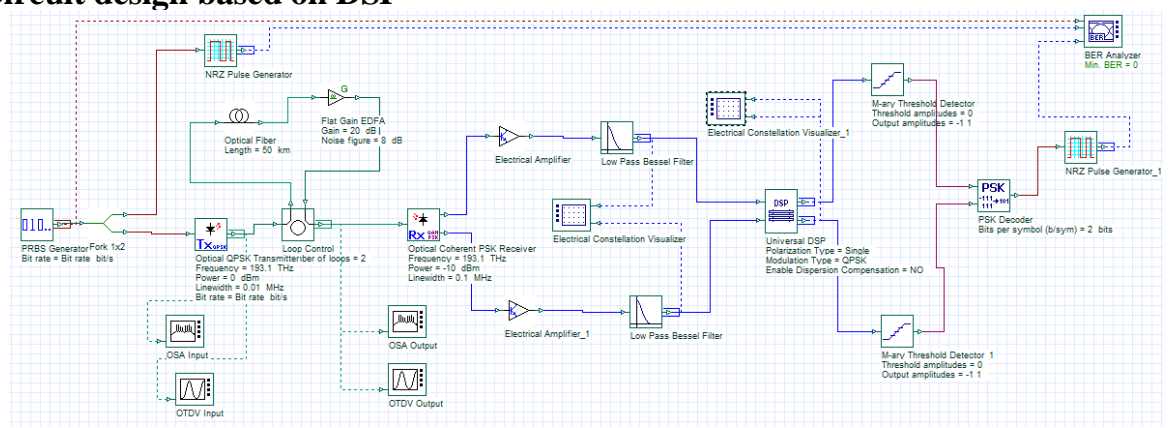

Fig.8.Circuit diagram 
Figure 8 is the design drawing of QPSK coherent optical communication system.The parameters of the simulation system are set as follows: the transmitter uses an optical rectilinear phase-shift coded transmitter with a frequency of $193.1 \mathrm{THz}$, and passes through a $50 \mathrm{~km}$ fiber, which is amplified by an amplifier, with a gain of $20 \mathrm{~dB}$ and a noise of $8 \mathrm{~dB}$.The receiver uses coherent detection, optical coherent PSK receiver, passes through the amplifier and the low pass filter, passes through the DSP module, obtains the constellation chart of this system.Compared with the previous design drawing, in order to realize long distance stable transmission, DSP module was added to carry out digital signal processing and reduce bit error rate.Advanced digital signal processing (DSP) algorithms have been implemented at receivers to compensate for signal damage during propagation ${ }^{[13]}$.

\subsection{The results demonstrate}

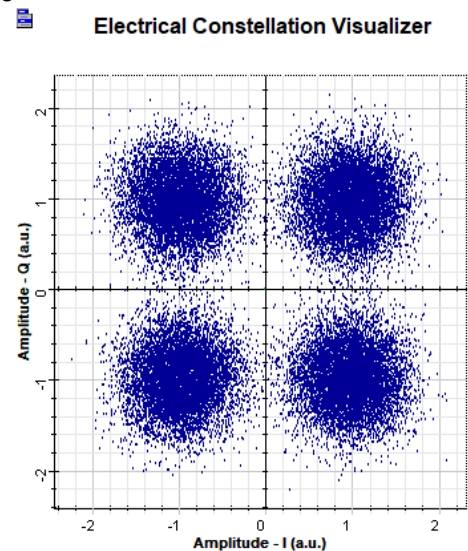

Compared with the constellation chart without DSP module, this chart is more complete than it.As can be seen from the figure, the actual signal distribution in the signal space is very concentrated, and the bit error rate is relatively low at this time.The design of DSP processing unit can reduce the bit error rate under QPSK modulation and improve the transmission efficiency of coherent optical communication.

\section{Conclusion}

This paper mainly discusses and studies the coherent optical communication system, puts forward the experimental theoretical results, and verifies the theoretical analysis through the simulation experiment.In the introduction, the development trend and advantage of coherent optical communication and its broad development prospect are briefly introduced.The second chapter describes the coherent optical communication system in all aspects. The advantages and disadvantages of coherent optical communication system are introduced and analyzed. The structure principle and key technology of coherent optical communication and its broad application prospect are expounded.The third chapter is the focus of this paper. It is based on the second chapter to carry out experimental simulation and theoretical analysis.In order to reduce bit error rate, DSP processing unit core algorithm design, simulation and analysis. The paper has been basically completed here. Due to the lack of knowledge, there are many shortcomings in this paper.I hope the teacher can point out in time that if I can understand the content of my research after reading this article, this is the goal I want to achieve, and it is also the greatest affirmation and support for my work. 


\section{References}

[1] A Alphonse;G S Sanyal.Coherent Optical Fibre Communication Systems[J].IETE Technical Review.1986,Vol.3(No.8): 429-433.

[2] Zhihong Zhu. Design and Implementation of Coherent Optical Communication System [J]. the electronic world,2016(09):139-140.

[3] Coherent Optical OFDM for Long-haul Communication System[D]. Beijing University of Posts and Telecommunications, 2018.

[4] Xiangdong Lv,Xuerui Liang,Qianchen Yu,Weidong Ma. Research status and development trend of optical communication technology [J]. Telecom science,2019,35(02):70-78.

[5] Jiong zhang. Application and development trend of optical fiber technology in communication engineering [J]. Engineering research,2019,4(15):237-238.

[6] Lihong Niu,Liping Kong. On Coherent Optical Communication [J]. SME management and technology (Under the ten-day),2012(07):219-220.

[7] Jirong Shi,Lin Liu.2.5 Gbit/s Research on Coherent Optical Communication System [J]. Shanghai Electric Technology,2019,12(03):14-16.

[8] Pengxia Li,Xizheng Xi. Experimental study on QPSK modulation and demodulation in coherent optical communication system [J]. Laser technology,2019,43(04):127-132.

[9] Wenbo Guo. Design and Simulation of QPSK Communication System Based on Matlab[J].Information and communication,2019(11):211-212.

[10] Jagana Bihari Padhy,Bijayananda Patnaik. 100 Gbps multiplexed inter-satellite optical wireless communication system[J]. Springer US,2019,51(7). 16.

[11] Ming - Seng Kao;Jingshown Wu.Forward and backward Raman amplifications in coherent optical communication systems[J].Journal of the Chinese Institute of Engineers.1992,Vol.15(No.4): 359-365.

[12] Yaming Wu,Guangcheng Li. Design of 100G coherent optical module [J]. Value engineering,2012,31(18):181-182.

[13] Dhiman Kakati,Subhash C. Arya. A 640-Gbps, 15.2344-b/s/Hz full-duplex optical fiber/wireless single-channel coherent communication system using IQM-based DP-256QAM and DSP techniques[J]. Springer US,2020,39(1).

[14] Yang $\mathrm{Yu}$. Coherent optical communication and its applications [J]. Physical bulletin,2001(09):43-46. 